\title{
Exact Analysis of the Outage Probability in Multiple-User Mobile Radio
}

\author{
Jean-Paul M. G. Linnartz
}

\begin{abstract}
A novel mathematical method is presented to express the outage probability for a desired radio signal received from a mobile transmitter in the presence of multiple interfering signals with combined log-normal and Rayleigh fading. In contrast to previously reported analysis, this exact method avoids approximation of the pdf of the received powers of the various signals. This is useful in determining the spectrum efficiency and performance of (interference-limited) radio networks for highcapacity cellular telephony, two-way paging, packet radio and other mobile data networks.
\end{abstract}

\section{INTRODUCTION}

$\mathbf{I}^{\mathrm{r}}$

$\mathrm{N}$ MOBILE telephony, the quality of service is often expressed in terms of the probability of outage experienced by subscribers near the boundary of the service area of a base station. Due to limited spectrum availability, radio networks become more and more limited by mutual interference between users. Therefore, the outage probability is usually determined in terms of excessive cochannel interference, whereas limitations due to noise usually can be overcome by appropriately increasing all transmit powers.

In the analyses of the spatial spectral efficiency of cellular radio reported so far, two different methods due to Fenton [1]-[5] and to Schwartz and Yeh [6]-[8] have been used to estimate the probability distribution of the interference power accumulated from several log-normal signals. Both methods have the disadvantage that probability density functions (pdf's) are not determined exactly but are approximated by matching means and variances. The latter (and more accurate) method requires iteration for more than two signals, and so numerical computations can become time consuming. Superimposed Rayleigh fading of the contributing signals requires further approximation. In some papers [2], [4], [5], [8], fast fading of the joint interference has been modeled by a Rayleigh distribution. However, if signals add incoherently during some time interval sufficiently longer than the bit duration, the joint amplitude of accumulated Rayleigh-fading signals follows a Nakagami $m$-distribution [9], [10]; this differs significantly from the Rayleigh distribution, especially when the number of contributing signals becomes large. A more appropriate model, recently proposed by Prasad and Arnbak [11], [12], describes combined shadowing and Rayleigh fading of one individual signal as a log-normal distribution with increased variance, and a mean decreased by $1.5 \mathrm{~dB}$. Schwartz and Yeh's

Paper approved by the Editor for Random Access and Distributed Communications Systems of the IEEE Communications Society. Manuscript received October 3, 1989 .

The author was with Telecommunications and Traffic-Control Systems Group, Delft University of Technology, 2600 GA Delft, The Netherlands. He is now with the Department of EECS, University of California at Berkeley, Berkeley CA 94720.

IEEE Log Number 9105157 method [7] is subsequently applied for incoherent cumulation. Instead of these two steps of approximation, this present letter offers a method of calculating the outage probability without any approximations of the pdf. Since the principal part of the calculation is performed analytically, this technique is faster in terms of required computer time.

\section{Channel MODEL}

In mobile radio communication, the normalized area-mean power $p_{a i}$ received from a mobile subscriber (indicated by index $i$ ) at distance $r_{i}$ from the base station has the general form

$$
p_{a i}=r_{i}^{-\beta}
$$

with $\beta$ in the range 3 to 4 . Assuming shadowing to be superimposed on (1), the local-mean power $p_{l i}$ is log-normally distributed about the area-mean power (1), viz.,

$$
f_{p l i}\left(p_{l i} \mid r_{i}\right)=\frac{1}{\sigma p_{l i} \sqrt{2 \pi}} \exp \left\{-\frac{\left(\beta \ln r_{i}+\ln p_{l i}\right)^{2}}{2 \sigma^{2}}\right\}
$$

where $\sigma$ is the logarithmic standard deviation of the shadowing, expressed in natural units.

Multipath reception causes Rayleigh fading, with the instantaneous received power $p_{s i}$ being distributed exponentially about the local-mean power $p_{l i}$. The conditional pdf of the received instantaneous power $p_{s i}$, given the propagation distance $r_{i}$, becomes [13]

$$
\begin{aligned}
f_{P s i}\left(P_{s i} \mid r_{i}\right)= & \int_{0}^{\infty} \frac{1}{p_{l i}} \exp \left\{\frac{-p_{s i}}{p_{l i}}\right\} \\
& \cdot \frac{1}{\sqrt{2 \pi} \sigma p_{l i}} \exp \left\{\frac{-\left(\ln p_{l i}+\beta \ln r_{i}\right)^{2}}{2 \sigma^{2}}\right\} d p_{l i} .
\end{aligned}
$$

\section{Cochannel InTERfERence Probability}

In a cellular network, outage is mainly caused by the interference from $n$ active users in the nearest cochannel cells. Hence, following [9]

$$
\begin{gathered}
\operatorname{Prob}(\text { outage } \mid n)=\operatorname{Prob}\left(p_{s 0} / p_{s n}<z\right) \\
=1 \int_{z}^{\infty} d x \int_{0}^{\infty} f p_{s 0}(x y) f p_{s n}(y) y d y .
\end{gathered}
$$

Here, $p_{s 0}$ is the instantaneous power of the desired signal (with index 0 ), $p_{s n}$ is the instantaneous power of the joint 
interference signal $\left(p_{s n} \triangleq \Sigma p_{s i}\right)$, and $z$ is the protection ratio required for reliable operation of a given receiver. $x$ and $y$ are dummy variables. The conditional outage probability, given the local-mean power $p_{t 0}$ of the desired signal, is obtained by inserting the exponential distribution due to Rayleigh fading in (4). This gives

$$
\text { Prob(outage } \left.\mid n, p_{l 0}\right)=1-\int_{0}^{\infty} \exp \left\{-\frac{y z}{p_{l 0}}\right\} f p_{s n}(y) d y \text {. }
$$

The second term on the right-hand side of (5) equals the definition [14] of the characteristic function (or Laplace transform) of the pdf of the instantaneous joint interference power $p_{s n}$ in the point $v=z / p_{l 0}[15]$. For incoherent (power) addition of $n$ uncorrelated interference signals, the outage probability can be written on the form of a product 9 of $n$ Laplace images, viz.,

$$
\text { Prob(outage } \left.\mid n, p_{l 0}\right)=1-\mathbb{ף}_{i=1}^{n} L\left\{f_{p i}, z / p_{10}\right\}
$$

where $f p_{s i}$ is on the form of (3) for $1 \leq i \leq n$. The corresponding characteristic function is found from

$$
\begin{aligned}
L\left\{f p_{s i}, v\right\}= & \int_{0}^{\infty} \exp \{-v x\} \int_{0}^{\infty} \frac{1}{p_{l i}} \exp \left\{\frac{-x}{p_{l i}}\right\} \\
& \cdot \frac{1}{\sqrt{2 \pi} \sigma p_{l i}} \exp \left\{\frac{-\left(\ln p_{l i}+\beta \ln r\right)^{2}}{2 \sigma^{2}}\right\} d p_{l i} d x \\
= & \int_{0}^{\infty} \frac{1}{1+v p_{l i}} \frac{1}{\sqrt{2 \pi} \sigma p_{l i}} \\
& \cdot \exp \left\{-\frac{\left(\ln p_{l i}+\beta \ln r_{i}\right)^{2}}{2 \sigma^{2}}\right\} d p_{l i}
\end{aligned}
$$

Combining (6) and (7), the conditional outage probability becomes

$$
\begin{gathered}
\operatorname{Prob}\left\{\text { out } \mid n, p_{l 0}\right\}=1-\mathbb{\Phi}_{i=1}^{n} \int_{0}^{\infty} \cdot \frac{p_{l 0}}{p_{l 0}+z p_{l i}} \frac{1}{\sqrt{2 \pi} \sigma p_{l i}} \\
\cdot \exp \left\{\frac{-\ln ^{2} p_{l i} r_{i}^{\beta}}{2 \sigma^{2}}\right\} d p_{l i} .
\end{gathered}
$$

Averaging over the shadowing (2) experienced by the desired signal $(i=0)$, the outage probability becomes

$$
\begin{aligned}
& \operatorname{Prob}\{\text { outage } \mid n\}=1-\int_{0}^{\infty} \frac{1}{\sqrt{2 \pi} \sigma p_{l 0}} \exp \left\{\frac{-\ln ^{2} p_{l 0} r_{0}^{3}}{2 \sigma^{2}}\right\} \\
& {\left[\mathbb{\Upsilon}_{i=1}^{n} \int_{0}^{\infty} \frac{p_{l 0}}{p_{l 0}+z p_{l i}} \frac{1}{\sqrt{2 \pi} \sigma p_{l i}}\right.} \\
&\left.\cdot \exp \left(\frac{-\ln ^{2} p_{l i} r_{i}}{2 \sigma^{2}}\right) d p_{l i}\right] d p_{l 0} .
\end{aligned}
$$

After substituting the logarithmic integration variables

$$
y_{i}^{2} \triangleq \frac{\ln ^{2} p_{l i} r_{i}^{\beta}}{2 \sigma^{2}}
$$

this is written as

Prob $\{$ outage $\mid n\}=1-\int_{0}^{\infty} d y_{0} \frac{1}{\sqrt{\pi}} \exp \left\{-y_{0}^{2}\right\}$
.$\left[\mathbb{\Upsilon}_{i=1}^{n} \frac{1}{\sqrt{\pi}} \int_{-\infty}^{\infty} \frac{r_{i}^{\beta} \exp \left\{y_{0} \sigma \sqrt{2}\right\} \exp \left\{-y_{i}^{2}\right\}}{r_{i}^{\beta} \exp \left\{y_{0} \sigma \sqrt{2}\right\}+z r_{0}^{\beta} \exp \left\{y_{i} \sigma \sqrt{2}\right\}} d y_{i}\right]$.

This expression is an exact solution to the stated problem. For channels without shadowing $(\sigma=0)$, probability (11) reduces to a simple analytic expression, as, e.g., in [5], [9], [10], [15]. To obtain numerical results for $\sigma>0$, the Hermite polynomial method ([14] 25.4.46) is used twice, resulting in the outage probability being computed from the double sum

Prob $\{$ outage $\mid n\}=1-\frac{1}{\sqrt{\pi}} \sum_{l=1}^{m} w_{l} \boldsymbol{\Phi}_{i=1}^{n}$
$.\left[\frac{1}{\sqrt{\pi}} \sum_{k=1}^{m} w_{k} \frac{r_{i}^{\beta} \exp \left\{x_{l} \sigma \sqrt{2}\right\}}{r_{i}^{\beta} \exp \left\{x_{l} \sigma \sqrt{2}\right\}+z r_{0}^{\beta} \exp \left\{x_{k} \sigma \sqrt{2}\right\}}\right]+R_{m}$.

The weight factors $w_{k}$ at the sample points $x_{l}$ are tabulated in [14] for an $m$-point integration. Since the remainder $R_{m}$ is sufficiently small for $m$ in the range of 10-20 terms [14], the method embodied in (12) proves to be much faster than Schwartz and Yeh's method [6]: in the latter method at least 40 complicated terms of a series have to be considered for each contributing interfering signal [6].

\section{Results for Cellular Radio}

In the analysis of spectrum efficiency of cellular radio, it is commonly assumed that all harmful interferers are at an equal distance of about the re-use distance $R_{d}\left(r_{i} \equiv R_{d}\right.$ for $0 \leq i \leq n$ ). The product $\pi$ in (12) then reduces to taking the $n$-th power. Fig. 1 presents the outage probability at the cell boundary (at $r_{0}=R_{0}$ ) versus the normalized reuse distance $R_{u}$, defined as

$$
R_{u} \triangleq \frac{R_{d}}{R_{0}}
$$

Shadowing with an intensity of $6 \mathrm{~dB}(\sigma=1,38)$ is assumed; the protection ratio is $10 \mathrm{~dB}(z=10)$.

Fig. 2 shows outage probability versus standard deviation of the shadowing, for a protection ratio of $10 \mathrm{~dB}(z=10)$ and a normalized reuse distance of $10\left(R_{u}=10\right)$. It is to be concluded that the extended Schwartz and Yeh method [8], [11], [12] produces somewhat pessimistic outage probabilities (roughly $10 \%$ ) compared to our exact result (11). The accuracy of the approximate method was found to improve with increasing $\sigma$ but appears relatively independent of the number of signals $n$. 


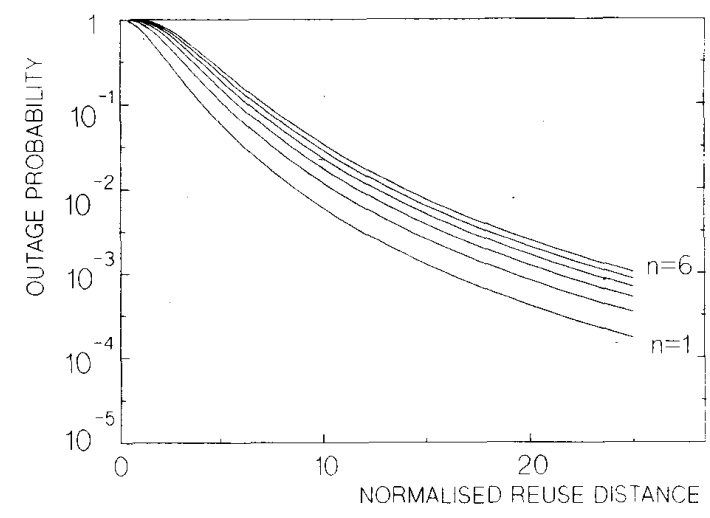

Fig. 1. Outage probability versus normalized reuse distance for a protection ratio of $10 \mathrm{~dB}$ and $n(n=1,2, \ldots, 6)$ cochannel interferers. The shadowing has a standard deviation of $\sigma=1.38(6 \mathrm{~dB})$.

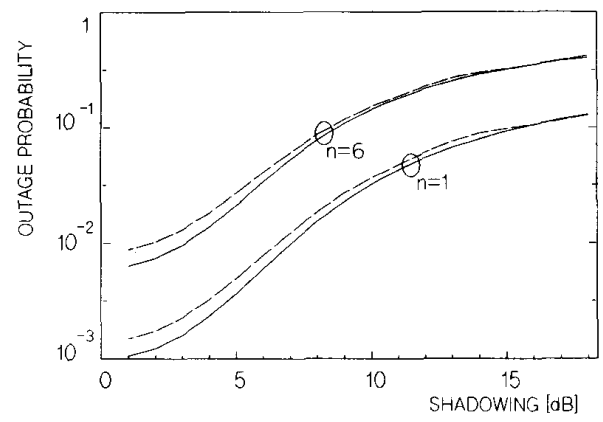

Fig. 2. Exact (11) (-) and approximate [12] (-) outage probability for one and six cochannel interferers, versus the standard deviation of the uncorrelated shadowing. The protection ratio is $10 \mathrm{~dB}$ and the normalized reuse distance of 10 .

\section{Discussion AND CONCLUSION}

A novel exact technique has been presented to compute outage probabilities due to interference in mobile radio channels with Rayleigh fading, shadowing and UHF groundwave propagation. While no closed-form solution has been found, the method leads to expressions in a form that can conveniently be used in numerical work. Although no analytic expression for the Laplace image of the log-normal distribution is known, the analysis is facilitated by the Laplace transformation of the pdf describing the combined Rayleigh fading and shadowing. The analytical expression (11), with the numerical result (12), can be used to improve accuracy and speed of calculations of spectrum efficiency in cellular radio [2]-[6], [8], and the throughput in slotted ALOHA with capture effects [9], [11]. Further, the method can be implemented in computerized design of real-world cell structures from a topological data base. In this event, highly accurate estimates of the area-mean power of all signals can be inserted in the final expression (11); the value of $\sigma$ should then be chosen appropriate to the resolution of the terrain data.

The approximation technique by Schwartz and Yeh [7] was hitherto considered [6], [8], [11] the most appropriate method available to cumulation of multiple log-normally fading signals. The method reported here requires no iterative and approximate analysis of moments of probability density functions in order to assess the pdf of cumulated signal powers. The new method is faster in numerical analysis than previous techniques. Further, no restriction is imposed on the standard deviation $\sigma$ of the shadowing fluctuations, whereas Fenton's method [1] is restricted to light shadowing (less than $4 \mathrm{~dB}$ ) and Schwartz and Yeh's method was developed for the range from 6 to $12 \mathrm{~dB}$ (though our investigation showed reasonable results outside this range).

The method proposed by Prasad and Arnbak [11], [12] to extend Schwartz and Yeh's approach, nevertheless, has the potential to model combined shadowing and Rician fading. The latter propagation model, which is appropriate to mobile radio outside the city centers, can presumably not be investigated by the method reported in this paper, since the useful property that the exponential power distribution of a Rayleigh fading signal can be interpreted as the Kernel of a Laplace transform [see (5) and (6)] is lost with Rician fading. Kernels containing Bessel functions as they occur with Rician fading, may not have the simple interpretation in terms of characteristic functions exploited in this correspondence.

\section{ACKNOWLEDGMENT}

The author is grateful to Prof. R. Prasad for fruitful discussions and comparing the presented method with alternative techniques. 


\section{REFERENCES}

[1] L. F. Fenton, "The sum of log-normal probability distributions in scatter transmission systems," IRE Trans. Commun. Syst., vol. CS-8, pp. 57-67, Mar. 1960.

[2] Y. Nagata and Y. Akaiwa, "Analysis for spectrum efficiency in single cell trunked and cellular mobile radio," IEEE Trans. Veh. Technol., vol. VT-35, pp. 100-113, Aug. 1987.

[3] D.C. Cox, "Cochannel interference considerations in frequency reuse small-coverage-area radio systems," JEEE Trans. Commun., vol. COM30, pp. 135-142, Jan. 1982.

[4] R. Muammar and S.C. Gupta, "Cochannel interference in highcapacity mobile radio systems," IEEE Trans. Commun., vol. COM-30, pp. 1973-1978, Aug. 1982.

[5] K. Daikoku and H. Ohdate, "Optimal channel reuse in cellular land mobile radio systems," IEEE Trans. Veh. Technol., vol. VT-32, pp. 217-224, Aug. 1983.

[6] R. Prasad and J.C. Ambak, "Comments on analysis for spectrum efficiency in single cell trunked and cellular mobile radio," IEEE Trans. Veh. Technol, vol. VT-36, Nov. 1988.

[7] S.C. Schwartz and Y.S. Yeh, "On the distribution function and moments of power sums with log-normal components," Bell Syst. Tech. J., vol. 61 , pp. $1441-1462$, Sept. 1982.

[8] R. Prasad, A. Kegel, and J.C. Arnbak, "Analysis of system performance of high-capacity mobile radio," in Proc. IEEE Veh. Techn. Conf. 1989 San Francisco, CA, May 3-5, 1989, pp. 306-309.

[9] J. C. Arnbak and W. van Blitterswijk, "Capacity of slotted-ALOHA in a Rayleigh fading channel," IEEE J. Select. Areas Commun., vol. SAC-5, pp. 261-269, Feb. 1987.

[10] J.-P. M. G. Linnartz and R. Prasad, "Threshold crossing rate and average non-fade duration in a Rayleigh-fading channel with multiple interferers," Archiv für Elektronik und Übertragungs-technik (AEÜ), vol. 43, no. 6, pp. 345-349, Nov./Dec. 1989.

[11] R. Prasad and J.C. Arnbak, "Effects of Rayleigh fading on packet radio channels with shadowing," in Proc. IEEE Tencon 1989, Bombay, India, Nov. 1989.

[12] R. Prasad and A. Kegel, "Improved assessment of interference limits in cellular radio performance," IEEE Trans, Veh. Technol, vol. 40, pp. 412-419, May 1991 .

[13] F. Hansen and F. Meno, "Mobile fading-Rayleigh and lognormal superimposed," IEEE Trans. Veh. Technol, vol. VT-26, pp. 332-335, Nov. 1977.

[14] Handbook of Mathematical Functions, M. Abramowitz and I. A. Stegun, Eds. New York: Dover, 1965

[15] D. Verhulst, M. Mouly, and J. Szpirglas, "Slow frequency hopping multiple access for digital cellular radiotelephone," IEEE J. Select. Areas Commun., vol. SAC-2, pp. 563-574, 1984. 